\title{
Temporal Trends and Predictors of Modern Contraceptive Use in Lusaka, Zambia, 2004-2011
}

\author{
Nancy L. Hancock, ${ }^{1,2}$ Carla J. Chibwesha, ${ }^{1,2}$ Marie C. D. Stoner, ${ }^{3}$ \\ Bellington Vwalika, ${ }^{4}$ Sujit D. Rathod, ${ }^{5}$ Margaret Phiri Kasaro, ${ }^{1,2}$ Elizabeth M. Stringer, \\ Jeffrey S. A. Stringer, ${ }^{1}$ and Benjamin H. Chi ${ }^{1}$ \\ ${ }^{1}$ Department of Obstetrics and Gynecology, University of North Carolina School of Medicine, 3009 Old Clinic Building, \\ Campus Box 7570, Chapel Hill, NC 27599-7570, USA \\ ${ }^{2}$ Centre for Infectious Disease Research in Zambia, 5032 Great North Road, P.O. Box 34681, 10101 Lusaka, Zambia \\ ${ }^{3}$ Department of Epidemiology, University of North Carolina School of Public Health, 2101 McGavran-Greenberg Hall, \\ CB No. 7435, Chapel Hill, NC 27599-7435, USA \\ ${ }^{4}$ Department of Obstetrics and Gynecology, University Teaching Hospital, P.O. Box 50110, Lusaka, Zambia \\ ${ }^{5}$ Department of Population Health, London School of Hygiene and Tropical Medicine, Keppel Street, London WC1E7HT, UK \\ Correspondence should be addressed to Carla J. Chibwesha; carla_chibwesha@med.unc.edu
}

Received 30 October 2015; Accepted 6 December 2015

Academic Editor: Renu Garg

Copyright (c) 2015 Nancy L. Hancock et al. This is an open access article distributed under the Creative Commons Attribution License, which permits unrestricted use, distribution, and reproduction in any medium, provided the original work is properly cited.

Introduction. Although increasing access to family planning has been an important part of the global development agenda, millions of women continue to face unmet need for contraception. Materials and Methods. We analyzed data from a repeated cross-sectional community survey conducted in Lusaka, Zambia, over an eight-year period. We described prevalence of modern contraceptive use, including long-acting reversible contraception (LARC), among female heads of household aged 16-50 years. We also identified predictors of LARC versus short-term contraceptive use among women using modern methods. Results and Discussion. Twelve survey rounds were completed between November 2004 and September 2011. Among 29,476 eligible respondents, 17,605 (60\%) reported using modern contraception. Oral contraceptive pills remained the most popular method over time, but use of LARC increased significantly, from less than $1 \%$ in 2004 to $9 \%$ by 2011 ( $p<0.001)$. Younger women (OR: 0.46, 95\% CI: 0.34, 0.61) and women with lower levels of education (OR: 0.70, 95\% CI: 0.56, 0.89) were less likely to report LARC use compared to women using short-term modern methods. Conclusions. Population-based assessments of contraceptive use over time can guide programs and policies. To achieve reproductive health equity and reduce unmet contraceptive need, future efforts to increase LARC use should focus on young women and those with less education.

\section{Introduction}

Family planning (FP) can help individuals and couples attain their desired number of children while providing benefits across a range of areas in women's reproductive health [1]. Since the 1994 International Conference on Population and Development, national governments, international agencies, and donor organizations have redoubled efforts to ensure that FP services are universally available in Sub-Saharan Africa and elsewhere $[2,3]$. In 2012 alone, it was estimated that 218 million pregnancies were prevented. In that same year, however, over 220 million women in the developing world still had an unmet need for contraception [4].

Currently, efforts to expand FP access have focused on increasing uptake of long-acting reversible contraception (LARC) $[5,6]$. Highly efficacious and safe, LARC includes both subdermal implants and intrauterine contraceptive devices (IUDs) [7]. These methods are user-independent, reversible, and discreet. They do not require recurrent visits to maintain efficacy and ensure a level of privacy $[7,8]$. Monitoring use of contraceptive methods, such as LARC, is critical to ongoing efforts to expand FP services. To date, 
however, most assessments have relied on sporadically and inconsistently collected data or older mathematical models $[9,10]$. Population-level field data are urgently needed to inform and optimize FP service implementation and policy.

\section{Materials and Methods}

We conducted a secondary analysis of a repeated crosssectional population-based survey to describe contraceptive use among women in Lusaka, Zambia. The original survey was designed to evaluate the impact of antiretroviral therapy scale-up on population-level mortality in a setting of high HIV prevalence. The methodology and primary outcomes of the survey have been detailed elsewhere [11, 12]. Briefly, between 2004 and 2011, we conducted 12 rounds of household surveys across Lusaka District, which includes Zambia's capital city Lusaka. From each of the district's 24 clinic catchments areas, 150 households were randomly sampled, for a total of 3,600 households per survey round. Fieldworkers asked a household member to identify the household head or heads. Interviewers were instructed to preferentially select female household heads because they would be more likely to recall details around household composition and events in the past 12 months. Household heads were questioned about sociodemographic characteristics, current contraceptive use or reasons for nonuse, health-related decision-making, HIV risk perception and testing history, and physical household characteristics and assets. Interviewers and participants completed all study procedures in English, Nyanja, or Bemba. Each study respondent provided written informed consent. This study was reviewed and approved by the ethical review committees from the University of Zambia Biomedical Research Ethics Committee (Lusaka, Zambia) and the University of North Carolina (Chapel Hill, NC, USA).

Our analysis was restricted to female household heads aged 16-50 years who reported not being pregnant, not being posthysterectomized, or not being postmenopausal. Male household heads were excluded from the analysis. Women who sought to delay or avoid pregnancy were asked about the specific methods they were using. Those who were not using any method of contraception were asked the main reasons behind this decision, and all reasons from each respondent were tabulated. In our analysis, contraceptive methods were categorized as modern versus traditional methods and, among those using modern contraception, we further categorized contraceptive methods into LARC or highly effective short-term reversible methods [7]. Modern contraception was defined as oral contraceptive pills (combination or progestin only), injectables (depot medroxyprogesterone acetate or norethisterone enanthate), subdermal implant, IUD, sterilization, condoms, supply methods (diaphragm, foam, or jelly), lactational amenorrhea method (LAM), or emergency contraception. LARC included subdermal implants and IUDs while highly effective short-term reversible contraception included oral contraceptive pills and injectables. LAM was excluded from the highly effective short-term reversible contraception because its effectiveness is limited to the first six months postpartum and duration of method use was not recorded for respondents. For women who reported using more than one method, they were analyzed according to the most effective method.

Descriptive statistics were used to compare characteristics between women using modern contraceptive versus those that were not. Percentages and frequencies or means with standard deviation were calculated via Chi-square or Wilcoxon tests and reported for categorical and continuous measures, as appropriate. The percentage of women using each individual method was calculated by survey year. For women not using contraception, reasons were tabulated by survey year. Among users of highly effective modern conceptive methods, logistic regression was used to create a predictive model for the odds of LARC versus shortterm contraception use (pills and injectables). We did not compare those who reported LARC with those who reported traditional or no method because we wanted to understand differences between those using highly effective modern contraception. All covariates with a $p$ value $<0.05$ were kept in the model using backwards stepwise regression. Survey year remained in the model regardless of $p$ value. Prevalence trends over time were calculated using linear regression and included chronological time in years as the independent variable. Statistical analyses were conducted with Stata 13.1 (StataCorp, College Station, TX) and figures were adjusted for the complex sampling design.

\section{Results and Discussion}

Between November 2004 and September 2011, a total of 12 survey rounds were completed across Lusaka District. Of the 43,200 heads of households who participated, 37,141 (86\%) were female. Among these, 7,665 women were excluded for the following reasons: being outside the 16-50-year age range $(n=2,963)$, report of pregnancy at time of survey $(n=3,511)$, and report of prior hysterectomy or onset of menopause $(n=1,191)$. The trend in pregnancy was nonlinear, with a low of $7 \%$ in 2004 and a high of $11 \%$ in 2008. Among the 29,476 respondents who were eligible for this analysis, 17,605 (60\%) reported using modern contraception (Table 1). Women using modern contraception were more likely to be under 30 years of age, be married, and have at least one child. They were also more likely to report prior HIV voluntary counseling and testing and perceive themselves at risk for HIV. Over the survey period, infrequent sex was the most frequently reported reason for not using modern contraception (45.9\%), followed by desire for pregnancy (23.4\%) and health concerns (14.9\%) (Figure 1).

Reported modern contraceptive use increased significantly between 2004 and 2011 (Figure 2). In 2004, 53\% of respondents endorsed using modern contraception with oral contraceptive pills being the most popular method (28\%) followed by LAM (18\%) and injectables (10\%). By 2011, 64\% of respondents reported using modern contraception. Oral contraceptive pills remained the most popular method (22\%), while the frequency of injectable use increased (19\%) and LAM use decreased (6\%). Condom use also increased over the survey period, from $4 \%$ to $7 \%$. LARC use was reported by less than $1 \%$ of respondents in 2004 but had increased to $9 \%$ by 2011 . Significant positive changes were observed 
TABLE 1: Demographic and socioeconomic characteristics of 16-50-year-old nonpregnant female heads of household by contraceptive status in Lusaka District, Zambia, 2004-2011.

\begin{tabular}{|c|c|c|c|}
\hline & $\begin{array}{l}\text { Women using modern } \\
\text { contraception } \\
(n=17,605)\end{array}$ & $\begin{array}{l}\text { Women not using modern } \\
\text { contraception } \\
(n=11,871)\end{array}$ & $p$ value \\
\hline Age, $\%$ & & & $<0.001^{1}$ \\
\hline $16-24$ & 26.9 & 18.8 & \\
\hline $25-30$ & 36.6 & 28.1 & \\
\hline $31-39$ & 28.3 & 29.2 & \\
\hline $40-50$ & 8.3 & 24.0 & \\
\hline Education, \% & & & $<0.001^{1}$ \\
\hline None & 3.4 & 5.1 & \\
\hline Primary & 38.9 & 39.2 & \\
\hline Secondary & 57.7 & 55.7 & \\
\hline Marital status, \% & & & $<0.001^{1}$ \\
\hline Married/cohabitating & 92.2 & 61.7 & \\
\hline Single/divorced/widowed & 7.8 & 38.3 & \\
\hline Religion, \% & & & $0.538^{1}$ \\
\hline Christian & 99.2 & 99.1 & \\
\hline Other & 0.8 & 0.9 & \\
\hline Living children, mean (SD) & $2.76(0.02)$ & $2.92(0.03)$ & $<0.001^{2}$ \\
\hline None & $4.1 \%$ & $17.2 \%$ & $<0.001^{1}$ \\
\hline$\geq 1$ & $95.9 \%$ & $82.8 \%$ & \\
\hline Socioeconomic status ${ }^{\mathrm{a}}, \%$ & & & $0.002^{1}$ \\
\hline Low & 40.5 & 42.2 & \\
\hline Medium & 39.3 & 36.6 & \\
\hline High & 20.2 & 21.2 & \\
\hline Household decision maker, \% & & & $<0.001^{1}$ \\
\hline Woman & 61.4 & 72.4 & \\
\hline Husband/male partner & 22.4 & 14.9 & \\
\hline Decision made jointly & 16.0 & 11.7 & \\
\hline Other & 0.3 & 1.0 & \\
\hline Voluntary counseling and testing, $\%$ & & & $<0.001^{1}$ \\
\hline Ever & 79.2 & 59.5 & \\
\hline Never & 20.8 & 40.5 & \\
\hline HIV knowledge ${ }^{\mathrm{b}}, \%$ & & & $<0.001^{1}$ \\
\hline All questions correct & 68.5 & 64.6 & \\
\hline Not all questions correct & 31.5 & 35.4 & \\
\hline HIV self-risk perception, $\%$ & & & $<0.001^{1}$ \\
\hline No risk & 36.3 & 47.7 & \\
\hline At risk & 50.7 & 37.8 & \\
\hline Unknown & 13.0 & 14.5 & \\
\hline Survey year, \% & & & $<0.001^{1}$ \\
\hline 2004 & 7.8 & 10.1 & \\
\hline 2005 & 15.1 & 20.1 & \\
\hline 2006 & 8.0 & 9.0 & \\
\hline 2007 & 26.3 & 25.0 & \\
\hline 2008 & 8.4 & 8.1 & \\
\hline 2010 & 8.9 & 6.6 & \\
\hline 2011 & 25.6 & 21.2 & \\
\hline
\end{tabular}

${ }^{1} \chi^{2}$ test.

${ }^{2}$ Wilcoxon test.

${ }^{a}$ The household wealth index was created using principal components analysis based on asset variables, electricity, energy source, type of floor, number of rooms, and water and sanitation variables similar to the demographic and health surveys.

${ }^{\mathrm{b}}$ As in the 2007 Zambia Demographic and Health Survey, interviewees were considered to have comprehensive knowledge of HIV if they correctly answered five questions about HIV transmission risk and so indicated that they knew that HIV cannot be transmitted through mosquitoes, HIV cannot be transmitted by witchcraft, HIV transmission risk can be reduced through condom use, HIV transmission can be reduced by having one HIV-negative sex partner, and a healthy-looking person can have HIV. 


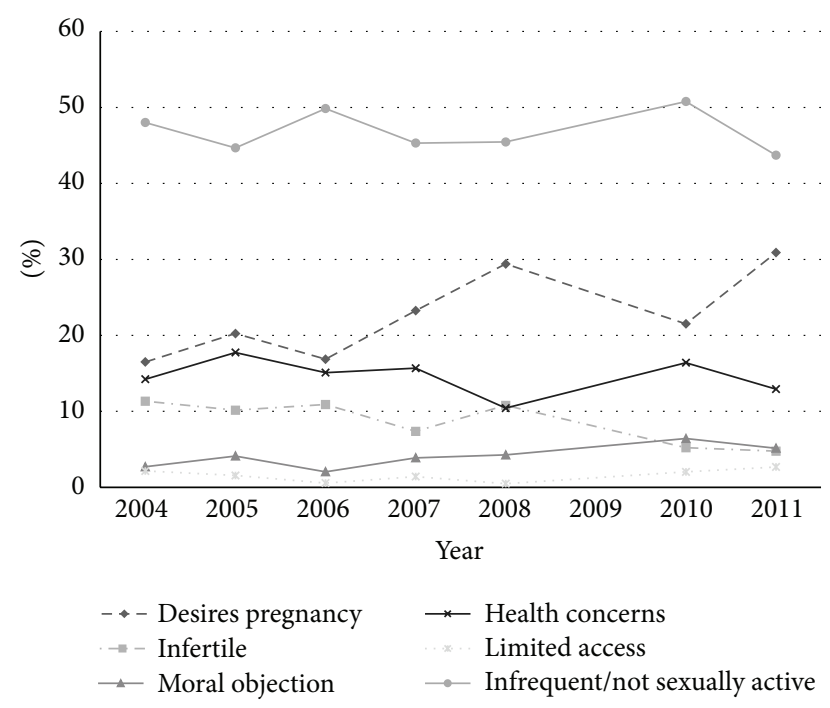

FIGURE 1: Reasons for nonuse of modern contraception among 16-50-year-old nonpregnant female heads of household in Lusaka District, Zambia, 2004-2011.

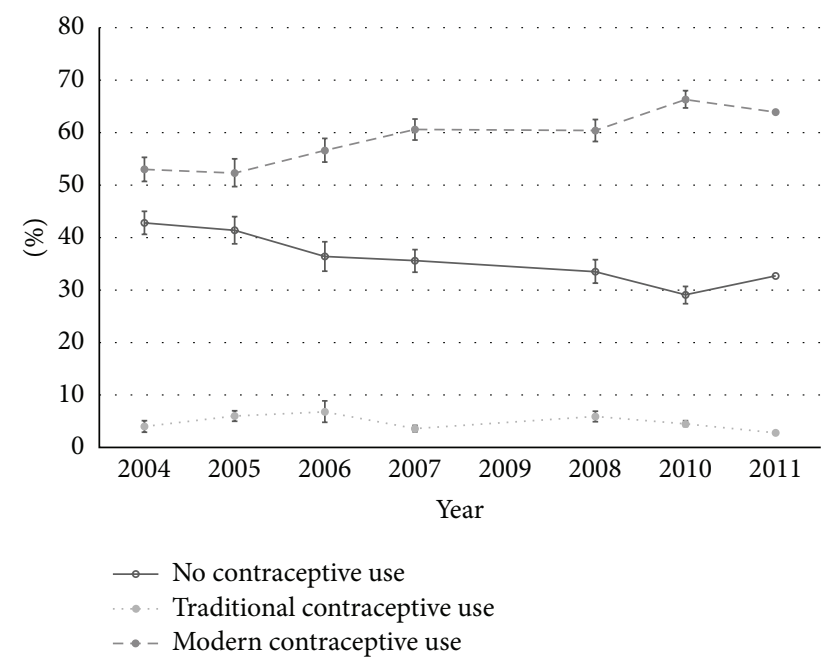

Figure 2: Trends in contraceptive use among 16-50-year-old nonpregnant female heads of household in Lusaka District, Zambia, 2004-2011.

for injectable, condom, and LARC use (Table 2). The greatest increases in LARC use were observed in the final two years of the survey (Figure 3).

Among respondents endorsing modern contraceptive use, 12,964 women reported highly effective reversible contraception use (74\%, Table 3). Of these, 12,098 (93\%) reported use of a short-term method (pills or injectables) and 866 (7\%) reported use of LARC. Factors associated with LARC use included having at least one child, having a higher socioeconomic status, and participating in the survey in 2010 and 2011. Younger women and those with less education were less likely to report LARC use. Marital status, religion, and household decision maker were not predictive of highly effective reversible contraception use (Table 3 ).

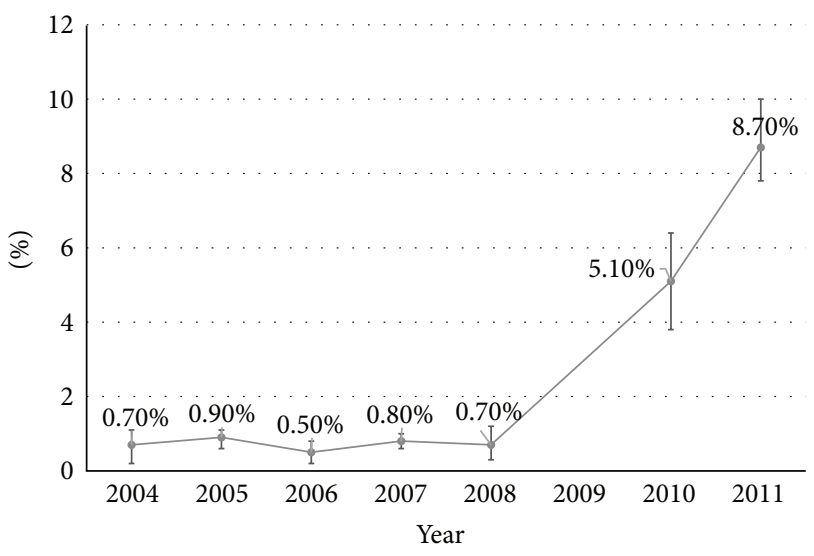

FIgURE 3: Trends in long-acting reversible contraceptive use among 16-50-year-old nonpregnant female heads of household in Lusaka District, Zambia, 2004-2011.

In this citywide repeated cross-sectional survey, we observed significant increases in modern contraceptive use generally and LARC use specifically over an eight-year period. Predictors of LARC use included older age, higher socioeconomic status, and survey year. Our findings suggest that programmatic efforts were highly successful in expanding FP services across Lusaka from 2004 to 2011, particularly in their promotion of LARC prior to 2010-2011. These population-level results are reassuring and consistent with increases in contraception uptake observed within specific health facilities [13].

We noted increased use of modern contraception over the observation period, a trend consistent with populationbased surveys in Zambia. In the 2007 Zambia Demographic and Health Survey (DHS), for example, 25\% of women were using a modern method, with pills, injectables, and LAM most commonly reported. Less than $1 \%$ reported LARC use [14]. By 2013-14, nearly one-third of female respondents reported using modern contraception, with injectables, pills, and subdermal implants being the most popular methods. More than 5\% reported LARC use countrywide, but condom use remained steady at $4 \%$ [15]. Similar results have been shown in East Africa [10]. We observed much higher modern contraceptive use, from a baseline prevalence of 53\% in 2004 to $64 \%$ in 2011 . This is likely due to greater resources and higher concentration of FP initiatives in the nation's capital city, as compared to more rural and remote parts of the country, including programs focused on expanding FP access to HIV-infected women and their partners [16-19].

Perhaps the most noteworthy finding from this secondary analysis was the increase in LARC use over the final rounds of the survey. Although this study was designed to measure impact of an HIV treatment program, rather than a specific contraceptive roll-out, we believe that the major contributor to this increased coverage was a highly successful initiative led by the Zambian Ministry of Health $(\mathrm{MOH})$ and the Society for Family Health (SFH). In an effort to rapidly expand services for LARC counseling and placement, this program trained and posted dedicated health providers in over 20 public health facilities starting November 2008 [13]. Over 
TABLE 2: Trends in contraceptive use and specific contraceptive method use among 16-50-year-old nonpregnant female heads of household in Lusaka District, Zambia, 2004-2011.

\begin{tabular}{|c|c|c|c|c|c|c|c|c|}
\hline & \multicolumn{7}{|c|}{ Survey year \% (95\% CI) } & \multirow{2}{*}{$\begin{array}{l}\text { Linear trend } \\
\text { coefficient, } p \text { value }\end{array}$} \\
\hline & 2004 & 2005 & 2006 & 2007 & 2008 & 2010 & 2011 & \\
\hline No contraceptive use & $\begin{array}{c}42.8 \% \\
(40.6,45.0)\end{array}$ & $\begin{array}{c}41.4 \% \\
(38.8,44.0)\end{array}$ & $\begin{array}{c}36.4 \% \\
(33.6,39.2)\end{array}$ & $\begin{array}{c}35.6 \% \\
(33.4,37.7)\end{array}$ & $\begin{array}{c}33.5 \% \\
(30.8,36.1)\end{array}$ & $\begin{array}{c}29.1 \% \\
(26.9,31.4)\end{array}$ & $\begin{array}{c}32.7 \% \\
(31.0,34.3)\end{array}$ & $\begin{array}{l}-1.6(-1.9,-1.2) \\
<\mathbf{0 . 0 1}\end{array}$ \\
\hline Traditional contraceptive use & $\begin{array}{c}4.0 \% \\
(2.9,5.1)\end{array}$ & $\begin{array}{c}6.0 \% \\
(5.0,7.0)\end{array}$ & $\begin{array}{c}6.8 \% \\
(4.8,8.9)\end{array}$ & $\begin{array}{c}3.6 \% \\
(3.0,4.2)\end{array}$ & $\begin{array}{c}5.9 \% \\
(4.8,7.0)\end{array}$ & $\begin{array}{c}4.5 \% \\
(3.5,5.5)\end{array}$ & $\begin{array}{c}2.8 \% \\
(2.3,3.4)\end{array}$ & $\begin{array}{l}-0.4 \%(-0.5,-0.2) \\
<\mathbf{0 . 0 1}\end{array}$ \\
\hline Modern contraceptive use & $\begin{array}{c}53.0 \% \\
(50.7,55.3)\end{array}$ & $\begin{array}{c}52.3 \% \\
(49.7,55.0)\end{array}$ & $\begin{array}{c}56.6 \% \\
(54.4 \\
58.9)\end{array}$ & $\begin{array}{c}60.6 \% \\
(58.6 \\
62.6)\end{array}$ & $\begin{array}{c}60.4 \% \\
(57.4,63.5)\end{array}$ & $\begin{array}{c}66.3 \% \\
(64.2,68.4)\end{array}$ & $\begin{array}{c}63.9 \% \\
(62.3,65.6)\end{array}$ & $\begin{array}{l}1.9 \%(1.5,2.3) \\
<\mathbf{0 . 0 1}\end{array}$ \\
\hline Condoms & $\begin{array}{c}3.5 \% \\
(2.5,4.6)\end{array}$ & $\begin{array}{c}4.5 \% \\
(3.7,5.3)\end{array}$ & $\begin{array}{c}5.3 \% \\
(4.4,6.3)\end{array}$ & $\begin{array}{c}6.2 \% \\
(5.4,7.0)\end{array}$ & $\begin{array}{c}5.8 \% \\
(4.7,7.0)\end{array}$ & $\begin{array}{c}7.0 \% \\
(5.6,8.5)\end{array}$ & $\begin{array}{c}6.7 \% \\
(5.7,7.8)\end{array}$ & $\begin{array}{l}0.4 \%(0.2,0.6) \\
<\mathbf{0 . 0 1}\end{array}$ \\
\hline Lactational amenorrhea & $\begin{array}{c}10.3 \% \\
(8.1,12.5)\end{array}$ & $\begin{array}{c}10.2 \% \\
(8.9,11.5)\end{array}$ & $\begin{array}{c}10.6 \% \\
(7.8,13.4)\end{array}$ & $\begin{array}{c}11.8 \% \\
(10.5,13.1)\end{array}$ & $\begin{array}{c}12.5 \% \\
(10.1,14.9)\end{array}$ & $\begin{array}{c}7.9 \% \\
(6.4,9.3)\end{array}$ & $\begin{array}{c}6.3 \% \\
(5.1,7.4)\end{array}$ & $\begin{array}{l}-0.7 \%(-0.9,-0.4) \\
<\mathbf{0 . 0 1}\end{array}$ \\
\hline Oral contraceptive pills ${ }^{\mathrm{a}}$ & $\begin{array}{c}27.8 \% \\
(26.0,29.6)\end{array}$ & $\begin{array}{c}27.2 \% \\
(25.4,29.0)\end{array}$ & $\begin{array}{c}27.8 \% \\
(24.6,31.1)\end{array}$ & $\begin{array}{c}27.7 \% \\
(26.2,29.1)\end{array}$ & $\begin{array}{c}27.4 \% \\
(24.4,30.3)\end{array}$ & $\begin{array}{c}23.6 \% \\
(20.5,26.7)\end{array}$ & $\begin{array}{c}22.8 \% \\
(21.6,24.1)\end{array}$ & $\begin{array}{l}-0.8 \%(-1.1,-0.5) \\
\mathbf{0 . 0 8}\end{array}$ \\
\hline Injectables & $\begin{array}{c}9.6 \% \\
(8.2,11.0)\end{array}$ & $\begin{array}{c}8.5 \% \\
(7.3,9.8)\end{array}$ & $\begin{array}{c}11.6 \% \\
(9.4,13.7)\end{array}$ & $\begin{array}{c}13.7 \% \\
(12.5,14.9)\end{array}$ & $\begin{array}{c}13.4 \% \\
(11.1,15.7)\end{array}$ & $\begin{array}{c}21.5 \% \\
(19.2,23.8)\end{array}$ & $\begin{array}{c}18.6 \% \\
(17.5,19.7)\end{array}$ & $\begin{array}{l}1.6 \%(1.4,1.8) \\
<\mathbf{0 . 0 1}\end{array}$ \\
\hline $\begin{array}{l}\text { Long-acting reversible } \\
\text { contraception }^{\text {b }}\end{array}$ & $\begin{array}{c}0.7 \% \\
(0.2,1.1)\end{array}$ & $\begin{array}{c}0.9 \% \\
(0.6,1.1)\end{array}$ & $\begin{array}{c}0.5 \% \\
(0.2,0.8)\end{array}$ & $\begin{array}{c}0.8 \% \\
(0.6,1.0)\end{array}$ & $\begin{array}{c}0.7 \% \\
(0.3,1.2)\end{array}$ & $\begin{array}{c}5.1 \% \\
(3.8,6.4)\end{array}$ & $\begin{array}{c}8.7 \% \\
(7.8,10.0)\end{array}$ & $\begin{array}{l}1.3 \%(1.1,1.4) \\
<\mathbf{0 . 0 1}\end{array}$ \\
\hline Implant & $\begin{array}{c}0.0 \% \\
(0,0.3)\end{array}$ & $\begin{array}{c}0.1 \% \\
(0.0,0.2)\end{array}$ & $\begin{array}{c}0.1 \% \\
(0.0,0.2)\end{array}$ & $\begin{array}{c}0.1 \% \\
(0.0,0.2)\end{array}$ & $\begin{array}{c}0.0 \\
(0.0,0.0)\end{array}$ & $\begin{array}{c}0 \% \\
(0.0,0.0)\end{array}$ & $\begin{array}{c}4.1 \% \\
(3.3,4.9)\end{array}$ & $\begin{array}{l}0.6 \%(0.4,0.7) \\
<0.01\end{array}$ \\
\hline IUD & $\begin{array}{c}0.7 \% \\
(0.2,1.1)\end{array}$ & $\begin{array}{c}0.8 \% \\
(0.5,1.0)\end{array}$ & $\begin{array}{c}0.4 \% \\
(0.1,0.7)\end{array}$ & $\begin{array}{c}0.7 \% \\
(0.3,1.1)\end{array}$ & $\begin{array}{c}0.7 \% \\
(0.3,1.1)\end{array}$ & $\begin{array}{c}5.1 \% \\
(3.8,6.4)\end{array}$ & $\begin{array}{c}4.6 \% \\
(3.8,5.3)\end{array}$ & $\begin{array}{l}0.7 \%(0.6,0.8) \\
<0.01\end{array}$ \\
\hline Sterilization & $\begin{array}{c}0.5 \% \\
(0.0,0.9)\end{array}$ & $\begin{array}{c}0.8 \% \\
(0.4,1.0)\end{array}$ & $\begin{array}{c}0.6 \% \\
(0.1,1.1)\end{array}$ & $\begin{array}{c}0.4 \% \\
(0.2,0.5)\end{array}$ & $\begin{array}{c}0.5 \% \\
(0.1,0.8)\end{array}$ & $\begin{array}{c}0.9 \% \\
(0.4,1.3)\end{array}$ & $\begin{array}{c}0.6 \% \\
(0.3,0.9)\end{array}$ & $\begin{array}{l}0.0 \%(-0.0,0.1) \\
\mathbf{0 . 7 6}\end{array}$ \\
\hline Other & $\begin{array}{c}0.6 \% \\
(0.0,1.3)\end{array}$ & $\begin{array}{c}0.3 \% \\
(0.1,0.5)\end{array}$ & $\begin{array}{c}0.2 \% \\
(0.0,0.4)\end{array}$ & $\begin{array}{c}0.1 \% \\
(0.0,0.1)\end{array}$ & $\begin{array}{c}0.0 \% \\
(0.0,0.0)\end{array}$ & $\begin{array}{c}0.3 \% \\
(0.1,0.5)\end{array}$ & $\begin{array}{c}0.2 \% \\
(0.1,0.3)\end{array}$ & $\begin{array}{l}-0.0 \%(-0.1,0.1) \\
\mathbf{0 . 2 4}\end{array}$ \\
\hline
\end{tabular}

${ }^{\mathrm{a}}$ Oral contraceptive pills include both combination and progestin only pills.

${ }^{\mathrm{b}}$ Long-acting reversible contraception includes subdermal implants and intrauterine devices.

the first 14 months, 33,609 women had initiated a long-acting contraceptive method, including subdermal implants $(n=$ $22,079,66 \%)$ and IUD $(n=11,530,34 \%)$. In addition, the $\mathrm{MOH}$ and SFH introduced postpartum IUD insertion in Lusaka in 2009, offering an additional opportunity for LARC uptake [20]. That such a high proportion of women still reported use of these methods several years later (i.e., in 2010 and 2011) is highly encouraging and suggests that LARC use can be sustained.

We observed substantial changes in the contraceptive method mix reported by women over the eight-year period. Use of oral contraceptives and LAM decreased over time, while use of injectables and LARC increased, particularly in the final survey rounds. The monitoring of such trends is important from a program perspective and can help to ensure that contraceptive supply meets ongoing demand [21]. In places like Zambia, understanding the uptake of specific methods may also be important in light of recent controversies around hormonal contraception and HIV. Although the overall evidence remains weak, some studies have suggested that hormonal contraception may be associated with increased rates of HIV acquisition among HIV-uninfected women and accelerated disease progression among women already infected with HIV [22, 23]. Recent work has also suggested that, among HIV-infected women on efavirenz, an antiretroviral agent recommended as part of first-line HIV treatment [24], certain contraceptive methods may be rendered less effective because of drug-drug interactions [2528]. As new evidence emerges, population-level data about FP method use can help identify individuals who may need more specific method counseling and inform broader health policies around contraception.

Our study is not without limitations. First, there were several concerns inherent to the survey itself, which was primarily designed to measure all-cause mortality at a population level. Our reliance on self-identified female heads of household may have introduced selection biases, particularly around past obstetrical history and future fertility intension. Due to the nature of the questions, responses from male household heads were excluded altogether from this secondary analysis. In addition, our sampling frame, based on the year 2000 census, may not have fully represented the increasing population of urban Lusaka [12]. Second, we relied on participant self-report for our measures of contraceptive prevalence and allowed respondents to select more than one method. We did not ask about the duration of current FP method use or previously used methods, which limited our ability to further describe FP trends. We were also unable to determine the impact of method switching, from nonuse to use, from traditional to modern methods, or between 
TABLE 3: Predictors of long-acting reversible contraception use among 16-50-year-old nonpregnant female heads of household using highly effective reversible contraception in Lusaka, Zambia, 2004-2011 $(N=12,964)^{\$}$.

\begin{tabular}{|c|c|c|c|c|}
\hline & $\begin{array}{c}\text { Women using } \\
\text { long-acting reversible } \\
\text { contraception }^{+} \\
(n=866) \\
\text { Percentage }\end{array}$ & $\begin{array}{l}\text { Women using highly } \\
\text { effective short-term } \\
\text { reversible contraception^ } \\
(n=12,098) \\
\text { Percentage }\end{array}$ & $\begin{array}{l}\text { Univariable } \\
\text { OR (95\% CI) }\end{array}$ & $\begin{array}{l}\text { Multivariable } \\
\text { OR (95\% CI) }\end{array}$ \\
\hline \multicolumn{5}{|l|}{ Age $^{*}$} \\
\hline $16-24$ & $14.6 \%$ & $25.8 \%$ & $0.33(0.25,0.45)$ & $0.46(0.34,0.61)$ \\
\hline $25-30$ & $35.3 \%$ & $37.3 \%$ & $0.56(0.42,0.74)$ & $0.66(0.50,0.89)$ \\
\hline $31-39$ & $35.7 \%$ & $28.5 \%$ & $0.74(0.57,0.96)$ & $0.80(0.61,1.04)$ \\
\hline $40-50$ & $14.4 \%$ & $8.5 \%$ & 1 & 1 \\
\hline \multicolumn{5}{|l|}{ Education* } \\
\hline None & $2.8 \%$ & $3.3 \%$ & $0.70(0.42,1.18)$ & $0.97(0.57,1.65)$ \\
\hline Primary & $27.2 \%$ & $38.9 \%$ & $0.58(0.45,0.74)$ & $0.70(0.56,0.89)$ \\
\hline Secondary & $70.0 \%$ & $57.8 \%$ & 1 & 1 \\
\hline \multicolumn{5}{|l|}{ Marital status } \\
\hline Married/cohabitating & $95.0 \%$ & $94.8 \%$ & $1.03(0.72,1.48)$ & \\
\hline Single/divorced/widowed & $5.0 \%$ & $5.2 \%$ & 1 & \\
\hline \multicolumn{5}{|l|}{ Religion } \\
\hline Christian & $99.0 \%$ & $99.2 \%$ & $0.72(0.26,2 / 03)$ & \\
\hline Other & $1.0 \%$ & $0.8 \%$ & 1 & \\
\hline Living children, mean (SD) & $3.04(0.08)$ & $2.76(0.02)$ & $1.11(1.06,1.17)$ & \\
\hline None & $1.9 \%$ & $2.8 \%$ & & \\
\hline$\geq 1$ & $98.1 \%$ & $97.2 \%$ & & \\
\hline \multicolumn{5}{|l|}{ Socioeconomic status* } \\
\hline Low & $27.6 \%$ & $39.3 \%$ & 1 & 1 \\
\hline Medium & $42.9 \%$ & $40.5 \%$ & $1.51(1.20,1.90)$ & $1.02(0.82,1.25)$ \\
\hline High & $29.6 \%$ & $20.2 \%$ & $2.09(1.59,2.74)$ & $1.42(1.14,1.78)$ \\
\hline \multicolumn{5}{|l|}{ Household decision maker } \\
\hline Woman & $64.8 \%$ & $60.2 \%$ & 1 & \\
\hline Husband/male partner & $21.2 \%$ & $23.4 \%$ & $0.84(0.66,1.07)$ & \\
\hline Decision made jointly & $13.9 \%$ & $16.1 \%$ & $0.80(0.63,1.01)$ & \\
\hline Other & $0.0 \%$ & $0.03 \%$ & $0.17(0.02,1.29)$ & \\
\hline \multicolumn{5}{|l|}{ Survey year* } \\
\hline 2004 & $2.0 \%$ & $8.1 \%$ & $0.94(0.46,1.92)$ & $0.98(0.48,1.97)$ \\
\hline 2005 & $4.9 \%$ & $15.2 \%$ & $1.25(0.83,1.88)$ & $1.27(0.86,1.89)$ \\
\hline 2006 & $1.5 \%$ & $8.3 \%$ & $0.70(0.37,1.33)$ & $0.71(0.38,1.34)$ \\
\hline 2007 & $6.8 \%$ & $26.5 \%$ & 1 & 1 \\
\hline 2008 & $2.0 \%$ & $8.4 \%$ & $0.94(0.50,1.78)$ & $0.90(0.48,1.70)$ \\
\hline 2010 & $13.6 \%$ & $8.9 \%$ & $5.90(3.96,8.80)$ & $5.50(3.69,8.35)$ \\
\hline 2011 & $69.2 \%$ & $24.6 \%$ & $10.91(7.96,14.95)$ & $10.73(7.87,14.61)$ \\
\hline
\end{tabular}

Percentages and odds ratios adjusted for complex sampling

\$Survey data from all years were appended into a single data set.

${ }^{*}$ Included in multivariable model.

${ }^{+}$Long-acting reversible contraception includes subdermal implants and intrauterine devices.

${ }^{\wedge}$ Highly effective short-term reversible contraception includes oral contraceptive pills and injectables. Lactational amenorrhea was excluded because duration was not recorded as part of the survey.

various modern methods. As such, the observed increase in LARC could reflect a favorable substitution effect or a true increase in overall use. Third, it is difficult to attribute these trends to specific strategies or initiatives within Lusaka at the time of survey. More detailed information about service utilization would have greatly enhanced this analysis, including reasons why women selected one contraceptive method over another. Fourth, we provide only limited comparisons to contemporaneous DHS surveys in Zambia (i.e., 2007, 2013-2014). We noted small but important differences in our source populations that could make such comparisons difficult. When determining reasons for noncontraceptive use, for example, marital status, pregnancy, menopause, and history of hysterectomy were considered differently between 
our analysis and that of the DHS [29]. Finally, we recognize that the external validity of this study may be limited because our survey was only conducted in Lusaka. While we used liberal eligibility criteria for inclusion into the parent study, contraceptive use patterns likely vary between urban and rural locations in Zambia.

\section{Conclusions}

Population-based assessments of contraceptive use, like the one described in this report, provide important information to guide FP program optimization and expansion. Ongoing monitoring of contraceptive prevalence can help program managers and policy makers match the conceptive supply to ongoing demand. Properly designed surveys can also serve as an evaluative framework for assessing new and promising interventions to increase uptake and retention within FP programs. Although the optimal timing and intensity of such assessments are yet to be determined, they are a necessary component for program improvement and should be included in coordinated efforts to achieve the post-2015 development agenda.

\section{Disclaimer}

The contents of this paper are solely the responsibility of the authors and do not necessarily represent the official views of the funding agencies.

\section{Conflict of Interests}

The authors declare that there is no conflict of interests regarding the publication of this paper.

\section{Acknowledgments}

This study was supported by a multicountry grant to the Elizabeth Glaser Pediatric AIDS Foundation from the U.S. Centers for Disease Control and Prevention (cooperative agreement U62/CCU12354) through the President's Emergency Plan for AIDS Relief. Additional investigator salary or trainee support was provided by the National Institutes of Health (T32 HD075731, R24 TW007988, and R25TW009340). The authors thank the study respondents and staff for their time and the Zambian Ministry of Health for its ongoing support of implementation and outcomes research.

\section{References}

[1] J. Cleland, A. Conde-Agudelo, H. Peterson, J. Ross, and A. Tsui, "Contraception and health," The Lancet, vol. 380, no. 9837, pp. 149-156, 2012.

[2] M. Greene, S. Joshi, and O. Robles, By Choice, not by Chance: Family Planning, Human Rights, and Development, United Nations Population Fund (UNFPA), New York, NY, USA, 2012.

[3] World Health Organization, Ensuring Human Rights in the Provision of Contraceptive Information and Services: Guidance and Recommendations, World Health Organization, Geneva, Switzerland, 2014.
[4] S. Singh and J. E. Darroch, Adding It Up: Costs and Benefits of Contraceptive Services-Estimates for 2012, Guttmacher Institute, United National Population Fund (UNFPA), New York, NY, USA, 2012.

[5] S. A. Cohen, "London Summit puts family planning back on agenda, offers new lease on life for millions of women and girls," Guttmacher Policy Review, vol. 15, pp. 20-24, 2012.

[6] Family Planning 2020. Family Planning 2020, June 2015, http:// www.familyplanning2020.org/.

[7] World Health Organization Department of Reproductive Health and Research (WHO/RHR) and Johns Hopkins Bloomberg School of Public Health/Center for Communication Programs (CCP) Knowledge for Health Project, Family Planning: A Global Handbook for Providers (2011 Update), CCP, Baltimore, Md, USA; WHO, Geneva, Switzerland, 2011.

[8] T. D. Ngo, O. Nuccio, K. Reiss, and S. K. Pereira, Expanding Long-Acting and Permanent Contraceptive Use in Sub-Saharan Africa to Meet FP2020 Goals, Marie Stopes International, London, UK, 2013.

[9] J. W. Townsend, R. Sitruk-Ware, K. Williams, I. Askew, and K. Brill, "New strategies for providing hormonal contraception in developing countries," Contraception, vol. 83, no. 5, pp. 405-409, 2011.

[10] J. E. Darroch and S. Singh, “Trends in contraceptive need and use in developing countries in 2003, 2008, and 2012: an analysis of national surveys," The Lancet, vol. 381, no. 9879, pp. 17561762, 2013.

[11] M. J. Giganti, J. W. Levy, Y. Banda et al., "Methods and baseline results of a repeated cross-sectional survey to assess the public health impact of antiretroviral therapy in Lusaka, Zambia," American Journal of Tropical Medicine and Hygiene, vol. 82, no. 5, pp. 971-977, 2010.

[12] S. D. Rathod, B. H. Chi, T. Kusanthan et al., "Trends in allcause mortality during the scale-up of an antiretroviral therapy programme: a cross-sectional study in Lusaka, Zambia," Bulletin of the World Health Organization, vol. 92, no. 10, pp. 734-741, 2014.

[13] J. Neukom, J. Chilambwe, J. Mkandawire, R. K. Mbewe, and D. Hubacher, "Dedicated providers of long-acting reversible contraception: new approach in Zambia," Contraception, vol. 83, no. 5, pp. 447-452, 2011.

[14] Central Statistical Office (CSO), Ministry of Health $(\mathrm{MOH})$, Tropical Diseases Research Centre (TDRC), University of Zambia, and Macro International, Zambia Demographic and Health Survey 2007, Macro International, Calverton, Md, USA, 2009.

[15] Central Statistics Office (CSO) [Zambia], Ministry of Health $(\mathrm{MOH})$ [Zambia], and ICF International, Zambia Demographic and Health Survey 2013-2014, Central Statistical Office, Ministry of Health, and ICF International, Rockville, Md, USA, 2014.

[16] C. J. Chibwesha, M. S. Li, C. K. Matoba et al., "Modern contraceptive and dual method use among HIV-infected women in Lusaka, Zambia," Infectious Diseases in Obstetrics and Gynecology, vol. 2011, Article ID 261453, 8 pages, 2011.

[17] K. Grabbe, R. Stephenson, B. Vwalika et al., "Knowledge, use, and concerns about contraceptive methods among sero-discordant couples in Rwanda and Zambia," Journal of Women's Health, vol. 18, no. 9, pp. 1449-1456, 2009.

[18] K. E. Mark, J. Meinzen-Derr, R. Stephenson et al., "Contraception among HIV concordant and discordant couples in Zambia: a randomized controlled trial," Journal of Women's Health, vol. 16, no. 8, pp. 1200-1210, 2007. 
[19] A. Disha, M. Bwembya, E. Collins, P. Papworth, and E. Ronnow, "Zambia: family planning quantitative and qualitative logistics system assessment," Task Order 1, USAID_DELIVER PROJECT, Arlington, Va, USA, 2008.

[20] S. Prager, P. Gupta, J. Chilambwe et al., "Feasibility of training Zambian nurse-midwives to perform postplacental and postpartum insertions of intrauterine devices," International Journal of Gynecology and Obstetrics, vol. 117, no. 3, pp. 243-247, 2012.

[21] E. E. Seiber, J. T. Bertrand, and T. M. Sullivan, "Changes in contraceptive method mix in developing countries," International Family Planning Perspectives, vol. 33, no. 3, pp. 117-123, 2007.

[22] C. B. Polis, S. J. Phillips, K. M. Curtis et al., "Hormonal contraceptive methods and risk of HIV acquisition in women: a systematic review of epidemiological evidence," Contraception, vol. 90, no. 4, pp. 360-390, 2014.

[23] S. J. Phillips, C. B. Polis, and K. M. Curtis, "The safety of hormonal contraceptives for women living with HIV and their sexual partners," Contraception, 2015.

[24] World Health Organization, Consolidated Guidelines on the Use of Antiretroviral Drugs for Treating and Preventing HIV Infection, World Health Organization, Geneva, Switzerland, 2013.

[25] S. H. Perry, P. Swamy, G. A. Preidis, A. Mwanyumba, N. Motsa, and H. N. Sarero, "Implementing the Jadelle implant for women living with HIV in a resource-limited setting: concerns for drug interactions leading to unintended pregnancies," AIDS, vol. 28, no. 5, pp. 791-793, 2014.

[26] N. K. Landolt, N. Phanuphak, S. Ubolyam et al., "Efavirenz, in contrast to nevirapine, is associated with unfavorable progesterone and antiretroviral levels when coadministered with combined oral contraceptives," Journal of Acquired Immune Deficiency Syndromes, vol. 62, no. 5, pp. 534-539, 2013.

[27] S. Y. El-Ibiary and J. M. Cocohoba, "Effects of HIV antiretrovirals on the pharmacokinetics of hormonal contraceptives," European Journal of Contraception and Reproductive Health Care, vol. 13, no. 2, pp. 123-132, 2008.

[28] J. A. Robinson, R. Jamshidi, and A. E. Burke, "Contraception for the HIV-positive woman: a review of interactions between hormonal contraception and antiretroviral therapy," Infectious Diseases in Obstetrics and Gynecology, vol. 2012, Article ID 890160, 15 pages, 2012.

[29] MEASURE DHS/ICF International, Demographic and Health Surveys Methodology: Standard Recode Manual for DHS 6. Demographic and Health Survey Toolkit of Methodology for the MEASURE DHS Phase III (2008-2013), MEASURE DHS/ICF International, Washington, DC, USA, 2013. 


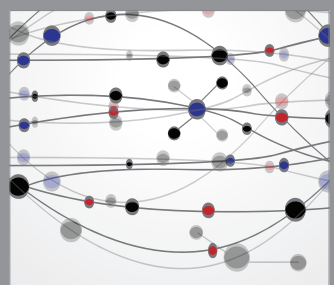

The Scientific World Journal
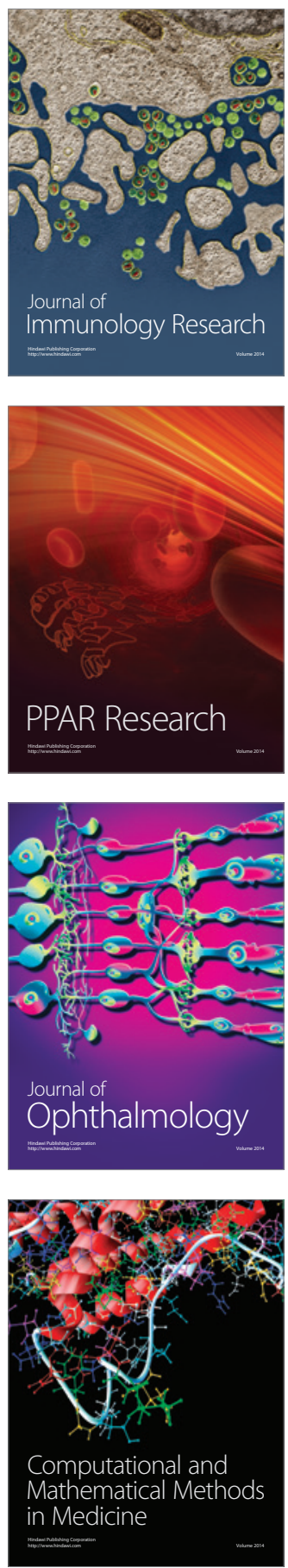

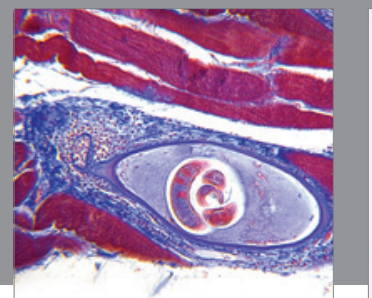

Gastroenterology

Research and Practice
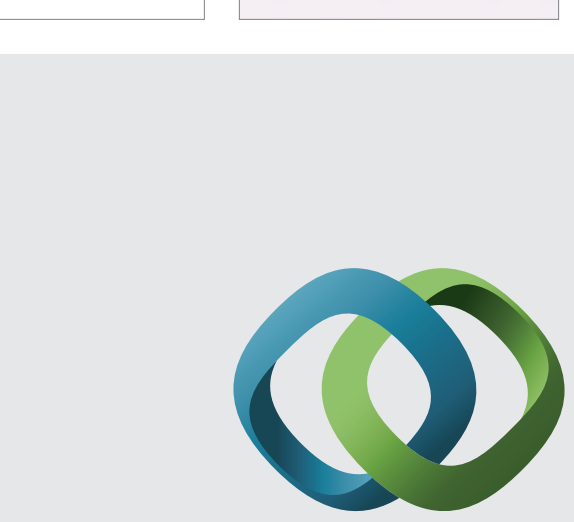

\section{Hindawi}

Submit your manuscripts at

http://www.hindawi.com
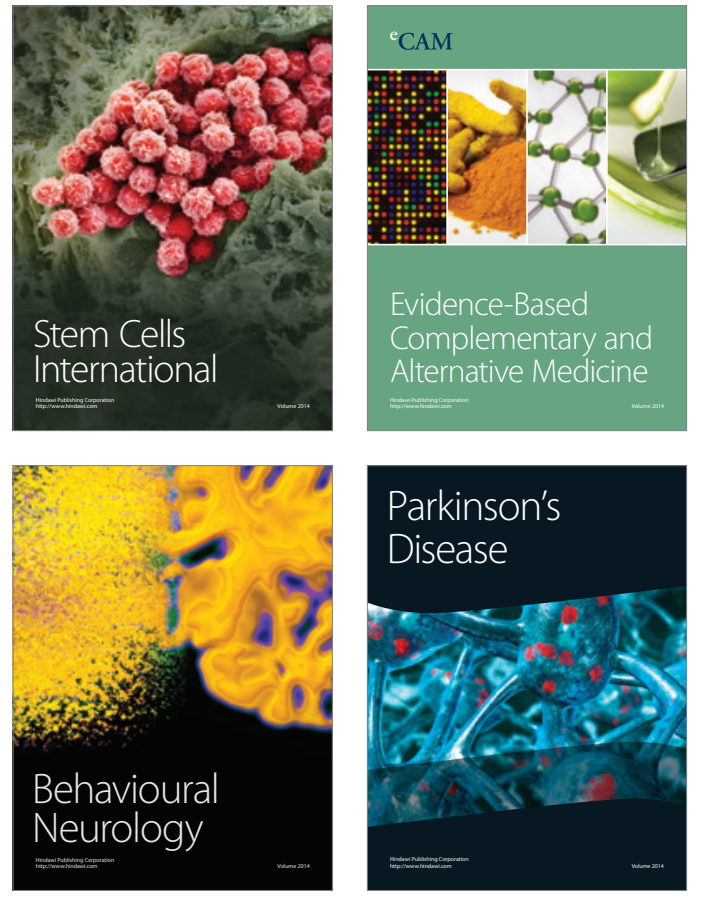
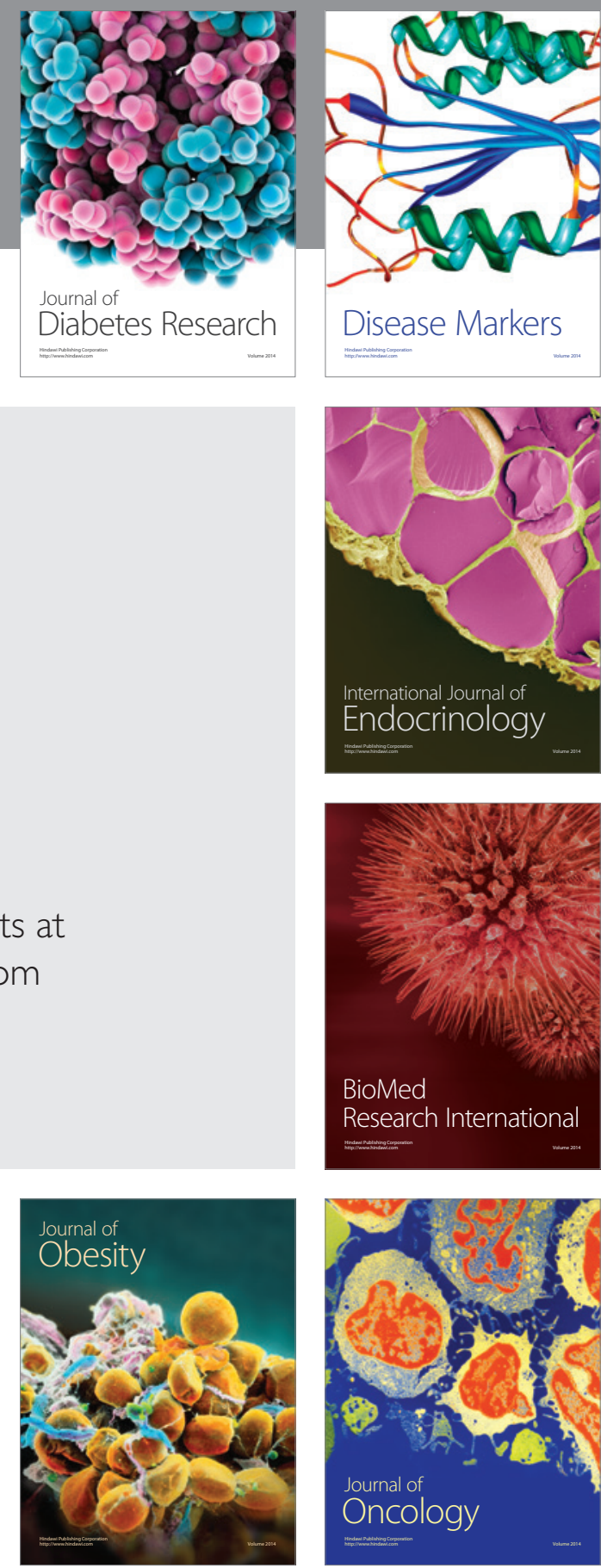

Disease Markers
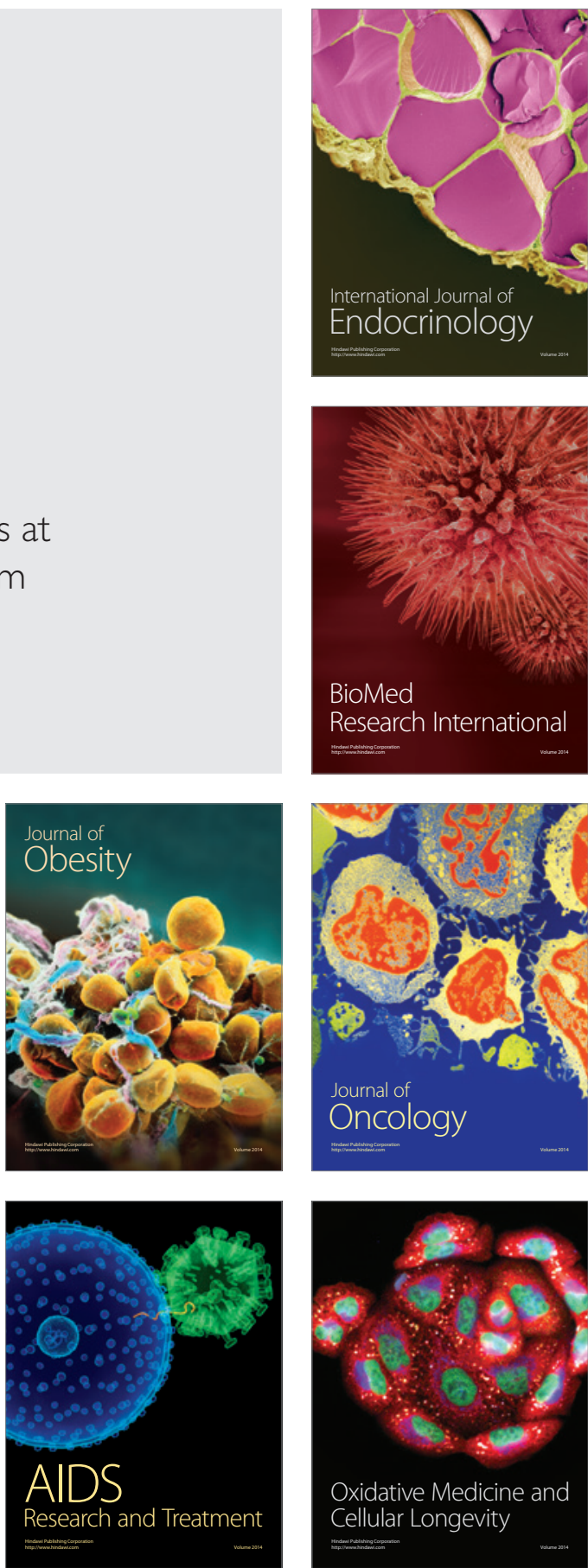